\title{
Management of Portal Hypertension in Children
}

\author{
Elizabeth Mileti • Philip Rosenthal
}

Published online: 7 October 2010

(C) The Author(s) 2010. This article is published with open access at Springerlink.com

\begin{abstract}
Management of portal hypertension in children has evolved over the past several decades. Portal hypertension can result from intrahepatic or extrahepatic causes. Management should be tailored to the child based on the etiology of the portal hypertension and on the functionality of the liver. The most serious complication of portal hypertension is gastroesophageal variceal bleeding, which has a mortality of up to $30 \%$. Initial treatment of bleeding focuses on stabilizing the patient. Further treatment measures may include endoscopic, medical, or surgical interventions as appropriate for the child, depending on the cause of the portal hypertension. $\beta$-Blockers have not been proven to effectively prevent primary or secondary variceal bleeding in children. Sclerotherapy and variceal band ligation can be used to stop active bleeding and can prevent bleeding from occurring. Transjugular intrahepatic portosystemic shunts and surgical shunts may be reserved for those who are not candidates for transplant or have refractory bleeding despite medical or endoscopic treatment.
\end{abstract}

\footnotetext{
E. Mileti

Pediatric Gastroenterology, Hepatology, and Nutrition, University of California, San Francisco,

500 Parnassus Avenue, Box 0136, MU 4-East,

San Francisco, CA 94143-0136, USA

e-mail: MiletiE@peds.ucsf.edu

P. Rosenthal $(\square)$

Pediatric Liver Transplant Program, Pediatric Hepatology,

University of California, San Francisco,

500 Parnassus Avenue, Box 0136, MU 4-East,

San Francisco, CA 94143-0136, USA

e-mail: prosenth@peds.ucsf.edu
}

Keywords Portal hypertension · Pediatric · Variceal hemorrhage $\cdot$ Variceal ligation · Sclerotherapy B Balloon tamponade $\cdot$ Transjugular intrahepatic portosystemic shunt (TIPS) · Surgical shunts · Octreotide · $\beta$-Blockers · Liver transplantation

\section{Introduction}

Portal hypertension is defined as portal vein pressures exceeding $5 \mathrm{~mm} \mathrm{Hg}$ or portal vein to hepatic vein gradient of greater than $10 \mathrm{~mm} \mathrm{Hg}$; variceal bleeding is associated with a gradient above $12 \mathrm{~mm} \mathrm{Hg}[1,2]$. When the portal system becomes congested, collateral pathways develop at the junctions between the high-pressure portal vessels and the low-pressure venous system, thereby forming varices. Variceal formations occur predominantly in the esophagus and in the stomach, retroperitoneally, and around the rectum and umbilicus (caput medusa). The most serious complication of portal hypertension is variceal bleeding, usually in the esophagus or stomach. Variceal hemorrhage is associated with a significant mortality rate of up to $30 \%$ [3]. Understanding the pathophysiology of portal hypertension is crucial to its diagnosis and management. Portal hypertension is caused by disruption of flow through the portal system and can be categorized into intrahepatic and extrahepatic etiologies. Extrahepatic causes can be further classified by prehepatic and posthepatic sites. Intrahepatic etiologies include presinusoidal, sinusoidal, or post-sinusoidal obstruction. Currently, portal hypertension in children most commonly results from an intrahepatic etiology, cirrhosis, or sinusoidal obstruction, which can occur with a multitude of liver diseases but predominantly with biliary atresia. Extrahepatic 
causes include obstruction of the portal vein, typically from instrumentation of the umbilical vein (usually during the neonatal period). Other etiologies include infection (eg. omphalitis), intra-abdominal abscess, severe dehydration, and blunt trauma to the abdomen. Additionally, BuddChiari syndrome, veno-occlusive disease, and cardiac disease can cause portal hypertension.

The natural history of variceal formation and bleeding in the presence of cirrhosis can occur fairly rapidly in children. A recent study of 139 children with biliary atresia found that $125(90 \%)$ showed signs of portal hypertension and underwent endoscopy at a median age of 13 months. Of those 125 children, $88(70 \%)$ had variceal formation. Gastrointestinal bleeding occurred in $20 \%$ of the 139 subjects at a median age of 17 months for the first bleed, with $75 \%$ of them bleeding prior to 2.5 years [4•].

\section{Diagnosis and Evaluation}

Portal hypertension is identified by a thorough history and physical examination. The history should focus on identifying factors that predispose the child to developing portal hypertension, such as family history of metabolic liver disease, personal history of hypercoagulable state, or history of umbilical vein instrumentation or abdominal infection. On examination, the majority of children with portal hypertension will have an enlarged spleen, unless other anomalies are present, such as asplenia or polysplenia (which can be seen in biliary atresia). Occasionally, ascites is present if the cause of portal hypertension is intrahepatic. The liver may be enlarged, but often is small and shrunken, and thus is an unreliable physical examination finding. Portal congestion can be seen rarely on physical examination as external or internal hemorrhoids and caput medusa. Imaging studies can also help confirm the presence of portal hypertension, including ultrasound with Doppler, contrast-enhanced $\mathrm{CT}$, and magnetic resonance angiography [5•]. Ultrasound can demonstrate heterogeneity of the liver in chronic liver disease, and Doppler examination provides information about portal vein patency and directionality of flow, both of which are important in the diagnosis and management of portal hypertension [5•].

Esophagogastroduodenoscopy (EGD) is the best mode to screen for esophageal and gastric varices and should be done once portal hypertension is suspected. However, a recent study by McKiernan et al. [6] showed that endoscopic ultrasound (EUS) was superior to visual examination by EGD in detecting early gastroesophageal varices in children with intestinal failure-associated liver disease. The study investigators evaluated 16 children, using upper endoscopy and EUS. Seven subjects were found to have gastroesophageal varices by EUS, although the varices were not seen on visual examination, indicating that EUS helps detect varices at an earlier stage than EGD [6]. Nevertheless, EGD has been important in detecting features associated with increased likelihood of bleeding, such as large tense varices, red spots, and red wale markings, information that is crucial for initiating treatment of an identified bleeding site [7, 8]. Additionally, the variceal grade identified and the presence of gastric varices were shown to be predictive of the risk of gastrointestinal bleed. The 5 -year risk of bleeding from biliary atresia patients is $59 \%$ with grade 3 varices, $17 \%$ with grade 2 varices, and $12 \%$ with no varices visible on initial endoscopy $[4 \bullet]$.

\section{Management of an Acute Variceal Bleed}

Acute gastrointestinal hemorrhage from variceal rupture in the esophagus or stomach often is a medical emergency, with 6 -week mortality rates approaching $30 \%$ in those with severe liver disease [3]. Children may have large-volume hematemesis or melena and may present in cardiovascular shock. Stabilizing the patient is the initial focus of management of gastrointestinal hemorrhage. Two largebore intravenous (IV) catheters must be placed immediately so that volume replacement with fluids and packed red blood cells can be delivered rapidly. When large-bore IV catheters cannot be established in children, intraosseous needles must be placed. Patients with coagulopathy from hepatic dysfunction and thrombocytopenia may require administration of IV vitamin $\mathrm{K}$, fresh frozen plasma, cryoprecipitate, factor VIIa, and/or platelets to help stop the bleeding. Nasogastric tube placement and gastric lavage are helpful in confirming that the site of bleeding is in the upper gastrointestinal tract. Additionally, they provide information about the extent of bleeding. Gastric lavage is usually done with room-temperature saline or sterile water and helps to evacuate the blood from the stomach, which allows for better visualization of the mucosa at the time of endoscopy. Initiation of an IV histamine ${ }_{2}$ receptor blocker or proton pump inhibitor helps to decrease the risk of bleeding from erosions or ulcerations. Once the child has been resuscitated, upper endoscopy should be performed to assess for the site of bleeding and initiate treatment, if indicated. Endoscopic treatment may include sclerotherapy, variceal banding, or injection of cyanoacrylate glue for gastric varices. Endoscopic treatments are discussed in detail below.

If bleeding cannot be controlled with the above measures, balloon tamponade with a Linton tube, Minnesota tube, or Sengstaken-Blakemore tube can be attempted. The Linton tube is a single-balloon tube used to stop the bleeding from gastric varices; the Minnesota and 
Sengstaken-Blakemore tubes have two balloons, one for the stomach for gastric varices, and the other for esophageal varices. The length of the esophageal balloon limits the use of the Minnesota and Sengstaken-Blakemore tubes to children weighing more than $40 \mathrm{~kg}$. Bleeding can be stopped in almost $90 \%$ of cases by balloon tamponade; however, the tubes are only temporizing measures and cannot be kept in place for more than $24 \mathrm{~h}$ [9]. Tube placement should be attempted only by individuals who have been trained and are comfortable with their use, because complication rates are high when the tubes are not properly placed. Complications include esophageal perforation, aspiration, mucosal ischemia, and airway obstruction $[9,10]$. When balloon tamponade is required, there should be a low threshold for intubation, and intubation is highly recommended.

\section{Pharmacologic Treatment}

The goal of pharmacologic intervention is to decrease portal pressure. The medications are classified into those that 1) stop active variceal bleeding, and 2) prevent variceal formation and variceal bleeding.

\section{Vasoactive Medications}

Vasoactive medications such as vasopressin, somatostatin, and octreotide are used to control active variceal bleeding. Vasopressin, the prototype drug in this group, increases splanchnic vascular tone and, as a result, decreases arterial splanchnic flow and decreases portal venous pressure. It is used as a continuous infusion of 0.002 to $0.005 \mathrm{U} / \mathrm{kg} /$ minute, to a maximum dose of $0.01 \mathrm{U} / \mathrm{kg} /$ minute. Although vasopressin was shown to control variceal hemorrhage in $53 \%$ of children, significant side effects are associated with vasoconstriction of the systemic blood pressure-causing ischemia to the bowel, heart, and kidneys - that make it undesirable for use [11]. Nitroglycerin has been used as a potent vasodilator to counter these effects, but this combination is not the most ideal for treatment of active variceal bleeding.

Somatostatin is a naturally occurring 14-amino acid peptide, and octreotide is an 8-amino acid synthetic analogue of somatostatin. Octreotide and somatostatin have the same pharmacologic effects, but octreotide has a longer half-life and therefore is used more often in the clinical setting [12]. Both medications decrease splanchnic blood flow and azygous blood flow and have less systemic vasoconstriction than vasopressin. The utility of octreotide or somatostatin in controlling variceal bleeding has been well studied in adults, but few studies have been performed in children. In 2001, Lam et al. [13] reported on the use of octreotide in variceal bleeding in three children. Another study reported on seven children with upper gastrointestinal bleeding (five from variceal bleeds) and found that bleeding stopped in six of the seven subjects who were given octreotide [14]. The largest retrospective series was described in 2004 by Eroglu et al. [15], who found that octreotide stopped gastrointestinal bleeding from portal hypertension in $71 \%$ of children. This cohort demonstrated a $52 \%$ post-treatment re-bleeding rate and mortality of $19 \%$. Octreotide dosing recommendations include a bolus of 1 to $2 \mu \mathrm{g} / \mathrm{kg}$ followed by a 1 to $2 \mu \mathrm{g} / \mathrm{kg} /$ hour infusion. The continuous infusion is titrated to effect. Once hemostasis is obtained, the dosing is tapered by $50 \%$ every $12 \mathrm{~h}$ for $24 \mathrm{~h}$, and then stopped [16]. Octreotide can be used to help stop or control an active bleed prior to endoscopy or when endoscopy cannot be performed.

\section{$\beta$-Blockers}

$\beta$-Blockers have no role in controlling acute gastrointestinal bleeding; however, they have been used in an attempt to prevent variceal formation and bleeding. Their role as a preventative has been well studied in adults. However, their use in children has been empirical and is based on case reports; no controlled or large studies have been done in children to support the effectiveness of $\beta$-blockers in preventing primary or secondary variceal bleeding.

Nonselective $\beta$-blockers have been used to reduce portal pressure and prevent primary and secondary bleeding episodes. They reduce portal hypertension by decreasing cardiac output and inducing splanchnic vasoconstriction through blockade of the $\beta-1$ and $\beta-2$ receptors [17]. In adult studies, $\beta$-blockers were shown to reduce the risk of variceal bleeding by about $50 \%$ [18]. In children, however, the benefits of $\beta$-blockade in reduction of primary or secondary bleeding are not clearly defined. The initial study of propranolol in children was published in 1985 [19]. Thirteen children with portal hypertension were given between 2 and $8 \mathrm{mg} / \mathrm{kg} /$ day of propranolol, with resultant decrease in heart rate of about $25 \%$ of baseline. Portal pressures as assessed by splenic pulp pressure were found to have been decreased by about $13 \%$ in children with compensated cirrhosis. No effect was seen in children with decompensated portal hypertension [19]. Regardless of the $13 \%$ decrease in those with compensated cirrhosis, the measurement of splenic pulp pressure has not been validated as a good predictive tool of portal hypertension.

Another report of $\beta$-blocker use for children with portal hypertension was a case series of 21 patients. Of the 21 children, 19 had cirrhosis and two had extrahepatic portal vein obstruction. Propranolol dosing was adjusted to achieve a $25 \%$ reduction in baseline heart rate. Thirtythree percent had a gastrointestinal hemorrhage during the 
follow-up period, median time of hemorrhage occurred at 3 years [20]. An additional study showed similar results, with bleeding rates of $15.6 \%$ in children taking propranolol for primary prophylaxis of variceal bleeding, and bleeding rates of $53 \%$ in those using the $\beta$-blocker for secondary prophylaxis [21]. These results show that even on propranolol, bleeding rates occurred at $15.6 \%$ to $33 \%$, which is not significantly improved from baseline bleeding rates seen in the natural history of this disease. Further, the use of $\beta$ blockers has a theoretical risk of harm from the $\beta-1$ receptor blockade causing decreased cardiac output, which may hinder appropriate heart rate increases during severe hemorrhage. At this point, $\beta$-blockers should not be routinely used in children as primary or secondary prophylaxis of variceal bleeding. Randomized, controlled trials are needed to evaluate the effects of nonselective $\beta$ blockers in children.

\section{Sclerotherapy and Variceal Ligation}

Sclerotherapy and variceal ligation provide an alternative option for treatment of actively bleeding varices and to help prevent variceal bleeding. Since the late 1980s, various studies have examined the use of sclerotherapy in children for extrahepatic and intrahepatic etiologies of portal hypertension, primarily as a means of stopping active bleeding and to obliterate varices entirely by using multiple treatments [22-31, 32•, 33-35]. The initial paper in 1988 by Howard et al. [22] retrospectively evaluated the outcomes of 108 children with variceal bleeding managed by injection sclerotherapy. Variceal obliteration was successful in $92 \%$ of those with extrahepatic portal hypertension and in $75 \%$ of those with intrahepatic portal hypertension. An additional 11 subjects with no prior history of bleeding were included, and sclerotherapy was used as a prophylactic technique in these children [22]. The other studies showed similar results; bleeding was stopped in about $92 \%$ to $95 \%$ of subjects, regardless of whether the bleeding had intrahepatic or extrahepatic etiologies. Additionally, sclerotherapy was shown to be useful even in very small children as young as 5 months and weighing only $5.5 \mathrm{~kg}$ [32•].

Despite the number of studies evaluating the role of sclerotherapy, only one randomized, controlled trial in children has looked at the value of endoscopic sclerotherapy on prevention of esophageal variceal hemorrhage. The study was performed in Brazil; 50 children in the sclerotherapy group received treatment every 21 days until the varices were obliterated, and 50 children in the control group received regular clinical and endoscopic examinations. After treatment was completed with sclerotherapy (mean 3.5 \pm 2.6 sessions), patients were followed for
18 months with endoscopic evaluations done every 6 months. The sclerotherapy group had only $6 \%$ esophageal variceal bleeding and $24 \%$ upper gastrointestinal bleeding during the trial, whereas the control group had $42 \%$ variceal bleeding and $48 \%$ upper gastrointestinal bleeding. However, mortality rates in both groups were the same, and the number of subjects with congestive hypertensive gastropathy increased in the treatment group [34].

Although endoscopic sclerotherapy has been used widely with effective treatment of bleeding, side effects from the sclerosant can be significant, such as perforation, bleeding, ulceration, and stricture formation at the injection site. Endoscopic variceal ligation (EVL) was subsequently developed and used in adults as an alternative treatment for esophageal varices, and was found to be superior to sclerotherapy in subjects with cirrhosis [35]. A randomized, controlled trial was published in 2002 evaluating the efficacy and safety of endoscopic sclerotherapy compared with endoscopic ligation in children. This study compared the two methods in 49 children with extrahepatic portal venous obstruction who had a history of bleeding varices. The EVL group included 25 subjects and the sclerotherapy group had 24 subjects. Variceal eradication was similar in the EVL group (96\%) and the sclerotherapy group (92\%). Variceal ligation, however, was able to eradicate the varices in fewer sessions than the sclerotherapy group $(3.9 \pm 1.1$ vs $6.1 \pm 1.7$, respectively, $P<0.0001$ ). Re-bleeding rates prior to variceal eradication were higher in the sclerotherapy group than in the EVL group $(25 \%$ vs $4 \%, P=0.049)$. Subjects in both groups were followed for about 22 months after variceal eradication. Recurrence rates of esophageal varices were not significantly different in the two groups (10\% in sclerotherapy group vs $17.4 \%$ in the EVL group) [36]. Another study investigated using EVL as a prophylactic measure to prevent first-time bleeding in children and adolescents with portal hypertension. Thirty-one children (aged 4 to 17 years) completed the study; 16 had portal hypertension from intrahepatic causes and 15 had portal hypertension from portal vein thrombosis. Of the 31 children, eradication of varices was achieved in 28 (90.3\%). No esophageal variceal bleeding occurred after or between treatments, and obliteration of the varices occurred on average after two to three treatments done at 3-month intervals. Two children developed hypertensive gastropathy, and one of them bled from this. Three children had recurrence of varices: one child with extrahepatic portal hypertension had varices recur at 13 months; the other two children, with intrahepatic portal hypertension, developed recurrent varices at 12 and 28 months [37].

Another therapeutic option very recently investigated in children is the use of cyanoacrylate glue for gastric varices [38]. This technique has been successfully used in adults for control of gastric varices $[38,39]$. A pilot study looked 
at the use of N-butyl-2-cyanoacrylate (Glubran 2; GEM Srl, Viareggio, Italy) in children younger than 2 years old and weighing less than $10 \mathrm{~kg}$ who had gastroesophageal varices. Seven of the 8 patients in the pilot study had active bleeding, and one patient had bled within 3 weeks prior to use of cyanoacrylate. Glue injection was successful in all infants, with 0.5 to $2 \mathrm{~mL}$ injected. Immediate control of bleeding was achieved in those with active bleeding. Rebleeding from gastric varices occurred in three of the eight patients $(37.5 \%)$, requiring a second treatment with cyanoacrylate. The use of cyanoacrylate glue clearly needs further investigation in infants and children, but thus far the results are promising for ability to stop active gastric variceal bleeding.

Despite the results of the above studies, esophageal variceal ligation cannot be performed in all children because of the size of the child's esophagus compared to the scope size and the associated ligature attachment, which can significantly obscure the view from the scope. The youngest child in studies where EVL was performed was 4 years old, but even infants as young as 5 months may require endoscopy and intervention for variceal formation and bleeding; in such circumstances, sclerotherapy can be crucial for treatment.

\section{Surgical Management and Transjugular Intrahepatic Portosystemic Shunts}

Esophageal variceal ligation, sclerotherapy, and medical treatments are useful in controlling variceal bleeding; however, these therapeutic measures do not treat the underlying cause. Transjugular intrahepatic portosystemic shunts (TIPS) and surgical shunts are the only means of helping to reduce portal pressure and in some cases definitively treat the underlying cause of portal hypertension. Candidates for TIPS or surgical shunts include children with extrahepatic portal vein obstruction, those with well-compensated cirrhosis who are unlikely to receive a transplant soon, and those with persistent variceal bleeding despite other management.

Transjugular intrahepatic portosystemic shunt (TIPS) is an interventional radiographic technique that places an intrahepatic metallic stent between the intrahepatic portions of the portal vein and the hepatic vein, thus creating a nonselective portocaval shunt. Because the shunt is nonselective, hepatic encephalopathy can occur. Other potential complications include shunt stenosis/thrombosis, bleeding, portal vein thrombosis, and migration of the stent into the right atrium. As with the other treatment modalities, it has been successfully used and studied in adults, but reports in children remain limited to case reports [40]. The limitations are generally related to the fact that many children with portal hypertension have cirrhosis because of biliary atresia and often have abnormal portal vein anatomy; moreover, the size of the child can lead to hepatic perforation and stent malposition. Despite the limitations and risks, TIPS is useful in variceal bleeding that is refractory to medical and endoscopic management, and can be a bridge to getting the patient to transplant. A recent study in adults by GarciaPagan et al. [41•] looked at use of TIPS in patients with cirrhosis and acute variceal bleeding, and randomly assigned them to early TIPS placement (within $72 \mathrm{~h}$ of hospitalization) or to medical treatment and EVL. In this particular patient population with a high risk for treatment failure (Child-Pugh class B with persistent bleeding or Child-Pugh class C), those who were placed in the early TIPS intervention group had improved mortality rates $(12 \%$ vs $38 \%$ ) and a reduction in treatment failure [41•].

Children with extrahepatic portal vein obstruction are particularly good candidates for surgical shunts. The shunts are divided into two groups: nonselective and selective shunts. The nonselective shunts, such as the mesocaval shunt and portocaval shunts, communicate with the entire portal system and have been associated with higher rates of hepatic encephalopathy compared with the selective shunts. One of the most common selective shunts is the distal splenorenal shunt (DSRS, Warren shunt). The DSRS, unlike the nonselective shunts, provides a way to decompress gastroesophageal varices via the short gastric veins and splenic vein while preserving the antegrade perfusion to the liver, which makes hepatic encephalopathy less likely. DSRS has been used successfully to improve severe thrombocytopenia and leukopenia [42]. The mesenteric-left portal shunt (Rex shunt) has also been used for portal vein thrombosis; it allows restoration of normal hepatopedal flow (which results in fewer neurologic effects). This shunt has been used to decompress portal hypertension in primary portal vein thrombosis and in portal vein thrombosis that has developed after liver transplant. The best outcomes have been described in patients in whom the internal jugular vein was used for the shunt [43].

Surgical shunts are usually reserved for treatment of portal hypertension when transplant is not an option; in patients with compensated cirrhosis in whom transplant is unlikely to occur soon; and in patients with multiple variceal bleeds that are refractory to both endoscopic and medical management.

\section{Conclusions}

Management of portal hypertension in children focuses on preventing and controlling variceal bleeding. Treatment in children has evolved over the past several decades as more studies have compared the efficacy of various medical, 
endoscopic, and surgical techniques in children. Although the adult studies are important and are often the basis for empiric therapy in children, the importance of randomized controlled trials to test these techniques in children cannot be overstated.

Conflicts of interest E. Mileti, none. P. Rosenthal has received fees and honoraria from Roche, Salix, GlaxoSmithKline, and Merck, and his institution has received grants from Roche and Bristol-Myers Squibb.

Open Access This article is distributed under the terms of the Creative Commons Attribution Noncommercial License which permits any noncommercial use, distribution, and reproduction in any medium, provided the original author(s) and source are credited.

\section{References}

Papers of particular interest, published recently, have been highlighted as:

- Of importance

1. Vargas HE, Gerber D, Abu-Elmagd K: Management of portal hypertension-related bleeding. Surg Clin North Am 1999, 79:122.

2. Garcia-Tsao G, Groszmann RJ, Fisher RL, et al.: Portal pressure, presence of gastroesophageal varices and variceal bleeding. Hepatology 1985, 5:419-424.

3. Carbonell N, Pauwels, Serfaty L, et al.: Improved survival after variceal bleeding in patients with cirrhosis over the past two decades. Hepatology 2004, 40:652-659.

4. - Duché M, Ducot B, Tournay E, et al.: Prognostic value of endoscopy in children with biliary atresia at risk for early development of varices and bleeding. Gastroenterology 2010 (Epub ahead of print). Duché et al. did a prospective evaluation of portal hypertension in children with biliary atresia. Their work demonstrated the significant risk of portal hypertension over the first years of life and the progression of varices.

5. Pariente D, Franchi-Abella S: Paediatric chronic liver diseases: how to investigate and follow up? Role of imaging in the diagnosis of fibrosis. Pediatr Radiol 2010, 40:906-919. This article is an excellent overview of various imaging techniques for chronic liver disease specifically in children.

6. McKiernan PJ, Sharif K, Gupte GL: The role of endoscopic ultrasound for evaluating portal hypertension in children being assessed for intestinal transplantation. Tranplantation 2008, 86:1470-1473.

7. Cales P, Zabotto B, Meskens C, et al.: Gastroesophageal endoscopic features in cirrhosis. Gastroenterology 1990, 98: 156-162.

8. North-Italian Endoscopic Club for the study and treatment of esophageal varices: Prediction of the first variceal hemorrhage in patients with cirrhosis of the liver and esophageal varices. A prospective multicenter study. N Engl J Med 1988, 319:983-989.

9. Sarin SK, Nundy S: Balloon tamponade in the management of bleeding oesophageal varices. Ann R Coll Surg Engl 1984, 66:30-32.

10. Pinto-Marques P, Romaozinho JM, Ferreira M, et al.: Esophageal perforation-associated risk with balloon tamponade after endoscopic therapy. Myth or reality? Hepatogastroenterology 2006, 53:536-539.
11. Tuggle DW, Bennett KG, Scott J, Tunnel WP: Intravenous vasopressin and gastrointestinal hemorrhage in children. J Pediatr Surg 1988, 23:627-629.

12. Tauber MT, Harris AG, Rochiccioli P: Clinical use of the long acting somatostatin analogue octreotide in pediatrics. Eur J Pediatr 1994, 153:304-310.

13. Lam JC, Aters S, Tobias JD: Initial experience with octreotide in the pediatric population. Am J Ther 2001, 8:409-415.

14. Siafakas C, Fox VL, Nurko S: Use of octreotide for the treatment of severe gastrointestinal bleeding in children. J Pediatr Gastroenterol Nutr 1998, 26:356-359.

15. Eroglu Y, Emerick KM, Whitington PF, Alonso EM: Octreotide therapy for control of acute gastrointestinal bleeding in children. $\mathrm{J}$ Pediatr Gastroenterol Nutr 2004, 38:41-47.

16. Lexi-Comp Online. Available at http://www.crlonline.com/crlsql/ servlet/crlonline. Accessed September 2010.

17. Feu F, Bordas JM, Luca A, et al.: Reduction of variceal pressure by propranolol: comparison of the effects of portal pressure and azygos blood flow in patients with cirrhosis. Hepatology 1993, 18:1082-1089.

18. D'Amico G, Pagliaro L, Bosch J: The treatment of portal hypertension: a meta-analytic review. Hepatology 1995, 22: 332-354.

19. Ozsoylu S, Kocak N, Yuce A: Propranolol therapy for portal hypertension in children. J Pediatr 1985, 106:317-321.

20. Shashidhar H, Langhans N, Grand RJ: Propranolol in prevention of portal hypertensive hemorrhage in children: a pilot study. J Pediatr Gastronterol Nutr 1999, 29:12-17.

21. Ozsoylu S, Kocak N, Demir H, et al.: Propranolol for primary and secondary prophylaxis of variceal bleeding in children with cirrhosis. Turk J Pediatr 2000, 42:31-33.

22. Howard ER, Stringer MD, Mowat AP: Assessment of injection sclerotherapy in the management of 152 children with oesphageal varices. Br J Surg 1988, 75:404-408.

23. Zargar SA, Yattoo GN, Javid G, et al.: Fifteen-year follow up of endoscopic injection sclerotherapy in children with extrahepatic portal venous obstruction. J Gastroenterol Hepatol 2004, 19: 139-145.

24. Dilawari JB, Chawla YK, Ramesh GN, et al.: Endoscopic sclerotherapy in children. J Gastroenterol Hepatol 1989, 4: $155-160$.

25. Yachha SK, Sharma BC, Kumar M, Khanduri A: Endoscopic sclerotherapy for esophageal varices in children with extrahepatic portal venous obstruction: a follow-up study. J Pediatr Gastroenterol Nutr 1996, 29:1-5.

26. Hill HD, Bowle MD: Endoscopic sclerotherapy for control of bleeding varices in children. Am J Gastroenterol 1991, 86: 472-476.

27. Sarin SK, Mirsa SP, Singal AK, et al.: Endoscopic sclerotherapy for varices in children. Am J Gastroenterol 1988, 7:662-666.

28. Stringer MD, Howard ER: Long-term outcome after injection sclerotherapy for esophageal varices in children with extrahepatic portal hypertension. Gut 1994, 35:257-259.

29. Horigome H, Nomura T, Saso K, et al.: Endoscopic injection sclerotherapy for esophagogastric variceal bleeding in children with biliary atresia. Hepatogastroenterology 1999, 46:30603062 .

30. Thapa BR, Mehta S: Endoscopic sclerotherapy of esophageal varices in infants and children. J Pediatr Gastroenterol Nutr 1990, $10: 430-434$.

31. Stringer MD, Howard ER, Mowat AP: Endoscopic sclerotherapy in the management of esophageal varices in 61 children with biliary atresia. J Pediatr Surg 1989, 24:438-442.

32. • Duché M, Habes D, Roulleau P, et al.: Prophylactic endoscopic sclerotherapy of large esophagogastric varices in infants with biliary atresia. Gastrointest Endosc 2008, 67:732-737. This is an 
important paper showing the effectiveness and feasibility of sclerotherapy in young infants (mean age of 13 months and mean weight of $8.2 \mathrm{~kg}$ ) with chronic liver disease.

33. Maksoud JG, Goncalves ME, Porta G, et al.: The endoscopic and surgical management of portal hypertension in children: analysis of 123 cases. J Pediatr Surg 1991, 26:178-181.

34. Goncalves ME, Cardoso SR, Maksoud JG: Prophylactic sclerotherapy in children with esophageal varices: long-term results of a controlled prospective randomized trial. J Pediatr Surg 2000, 35: 401-405.

35. Hou MC, Lin HC, Kuo BIT, et al.: Comparison of endoscopic variceal injection sclerotherapy and ligation for the treatment of esophageal hemorrhage: a prospective randomized trial. Hepatology 1995, 21:1517-1522.

36. Zargar SA, Javid G, Khan BA, et al.: Endoscopic ligation compared with sclerotherapy for bleeding esophageal varices in children with extrahepatic portal venous obstruction. Hepatology 2002, 36:666-672.

37. Celinska-Cedro D, Teisseyre M, Woynarowski M, et al.: Endoscopic ligation of esophageal varices for prophylaxis of first bleeding in children and adolescents with portal hypertension: preliminary results of a prospective study. J Pediatr Surg 2003, 38:1008-1011.

38. Rivet C, Robles-Medranda C, Dumortier J, et al.: Endoscopic treatment of gastroesophageal varices in young infants with cyanoacrylate glue: a pilot study. Gastrointest Endosc 2009, 69:1034-1038.

39. Lo GH, Lai KH, Cheng JS, et al.: A prospective, randomized trial of butyl cyanoacylate injection versus band ligation in the management of bleeding gastric varices. Hepatology 2001, 35:1060-1064.

40. Heyman MB, LaBerge JM, Somberg KA, et al.: Transjugular intrahepatic portosystemic shunts (TIPS) in children. J Pediatr 1997, 131:914-919.

41. - Garcia-Pagan JC, Caca K, Bureau C, et al.: Early use of TIPS in patients with cirrhosis and variceal bleeding. N Engl J Med 2010, 362:2370-2379. Garcia-Pagan et al. evaluated the use of TIPS in high-risk adults (Child-Pugh class B with persistent bleeding or Child-Pugh class C) randomly assigning them to early TIPS placement or EVL. They showed that those with early TIPS (within 72 hours of admission) had improved mortality rates and reduction in treatment failure.

42. Moon SB, Jung SE, Ha JW, et al.: The usefulness of distal splenorenal shunt in children with portal hypertension for the treatment of severe thrombocytopenia and leukopenia. World J Surg 2008, 32:483-487.

43. Krebs-Schmitt D, Briem-Richter A, Grabhorn E, et al.: Effectiveness of Rex shunt in children with portal hypertension following liver transplant or with primary portal hypertension. Pediatr Transplant 2009, 13:540-544. 